\title{
CrMAPK3 regulates the expression of iron-deficiency-responsive genes in Chlamydomonas reinhardtii
}

\author{
Xiaowen $\mathrm{Fei}^{1 \dagger}$, Junmei $\mathrm{Yu}^{2 \dagger}$, Yajun $\mathrm{Li}^{2}$ and Xiaodong Deng ${ }^{2^{*}}$
}

\begin{abstract}
Background: Under iron-deficient conditions, Chlamydomonas exhibits high affinity for iron absorption. Nevertheless, the response, transmission, and regulation of downstream gene expression in algae cells have not to be investigated. Considering that the MAPK pathway is essential for abiotic stress responses, we determined whether this pathway is involved in iron deficiency signal transduction in Chlamydomonas.

Results: Arabidopsis MAPK gene sequences were used as entry data to search for homologous genes in Chlamydomonas reinhardtii genome database to investigate the functions of mitogen-activated protein kinase (MAPK) gene family in $C$. reinhardtii under iron-free conditions. Results revealed 16 C. reinhardtii MAPK genes labeled CrMAPK2-CrMAPK17 with TXY conserved domains and low homology to MAPK in yeast, Arabidopsis, and humans. The expression levels of these genes were then analyzed through qRT-PCR and exposure to high salt (150 mM NaCl), low nitrogen, or iron-free conditions. The expression levels of these genes were also subjected to adverse stress conditions. The mRNA levels of CrMAPK2, CrMAPK3, CrMAPK4, CrMAPK5, CrMAPK6, CrMAPK8, CrMAPK9, and CrMAPK11 were remarkably upregulated under iron-deficient stress. The increase in CrMAPK3 expression was 43-fold greater than that in the control. An RNA interference vector was constructed and transformed into C. reinhardtii 2A38, an algal strain with an exogenous FOX1:ARS chimeric gene, to silence CrMAPK3. After this gene was silenced, the mRNA levels and ARS activities of FOX1:ARS chimeric gene and endogenous CrFOX1 were decreased. The mRNA levels of iron-responsive genes, such as CrNRAMP2, CrATX1, CrFTR1, and CrFEA1, were also remarkably reduced.
\end{abstract}

Conclusion: CrMAPK3 regulates the expression of iron-deficiency-responsive genes in C. reinhardtii.

Keywords: Chlamydomonas reinhardtii, Mitogen-activated protein kinases, Iron deficiency, Real-time PCR

\section{Background}

Chlamydomonas reinhardtii (Volvocales, Chlorophyta) is a single-celled eukaryotic and flagellated green alga, whose three genetic systems located in the nucleus, chloroplast, and mitochondria can be used for transformation. This alga is regarded as a "photosynthetic yeast" because of its easy culturing process, rapid growth, short life cycle, and high photosynthetic efficiency. With its three genome sequences, this model organism is highly useful for cell and molecular biology research [1].

\footnotetext{
* Correspondence: xiaodong9deng@hotmail.com

${ }^{\dagger}$ Equal contributors

${ }^{2}$ Institute of Tropical Bioscience and Biotechnology, Chinese Academy of Tropical Agricultural Science, Key Laboratory of Tropical Crop Biotechnology, Ministry of Agriculture, Haikou 571101, China

Full list of author information is available at the end of the article
}

In phosphorylation cascades, mitogen-activated protein kinases (MAPKs) are eukaryotic signal proteins involved in extracellular signal amplification and intracellular signal transduction in yeasts, animals, and plants [2-4]. Combined with other signal molecules, MAPKs transfer external stimuli via successive phosphorylation reactions: MAPKKKs $\rightarrow$ MAPKKs $\rightarrow$ MAPKs. Progressively and continuously enlarged signals, such as environmental stress factors, including high salinity, high osmotic pressure, and low temperature, reach the nucleus and regulate downstream gene expression [5, 6]. In eukaryotic cells, phosphorylation cascades are composed of MAPKs, MAPKKs, and MAPKKKs. Homo sapiens possesses 15 MAPKs, 7 MAPKKs, and 16 MAPKKKs, while Arabidopsis contains 20 MAPKs, 10 MAPKKs, and 80 MAPKKKs. Few MAPK cascades have been described because of 
the complexity of genetic networks and pleiotropic and interaction effects. MAPK genes have been identified in plants, such as Arabidopsis, rice, corn, wheat, and barley [7-13]. MAPKs function through stress-response pathways $[14,15]$.

Iron is an essential trace element for most living organisms. A precise iron regulation system is necessary to maintain the dynamic equilibrium of iron [16] because iron overload and deficiency cause metabolic disorders. Following nitrogen and phosphate deficiencies, iron deficiency restricts plant growth and yield and consequently induces crop chlorosis and yields low productivity. In humans, insufficient iron concentrations trigger iron deficiency anemia or iron deficiency syndrome. Iron has also been considered a growth-limiting factor in some tumor cells. Therefore, iron chelators are clinically used for cancer suppression.

Under iron-deficient conditions, Chlamydomonas exhibits high affinity for iron absorption that slightly differs from iron absorption in plants. Environmental ferric iron is reduced to ferrous iron via FRE1 (homology of Arabidopsis FRO2 [17]) on the plasma membrane and then putatively transferred to FOX1 by FEA1 [18]. Afterward, FOX1 oxidizes ferrous iron to ferric iron, which is then transported to the cytoplasm by FTR1 on the plasma membrane [19-21]. The expression of the genes encoding these proteins is significantly increased under iron-deficient conditions, and this phenomenon indicates that iron deficiency signals in these genes are regulated. Nevertheless, the response, transmission, and regulation of downstream gene expression in algal cells have yet to be investigated. Considering that the MAPK pathway is essential for nonbiological stress responses, we determined whether this pathway is involved in iron deficiency signal transduction in Chlamydomonas. In this study, Arabidopsis MAPKs were used to search for the corresponding genes in the Chlamydomonas genome database (https://phytozome.jgi.doe.gov/ pz/portal.html \#), and 16 homologous genes, namely, CrMA PK2-CrMAPK17, were obtained. The mRNA expression level variation of these genes exposed to different stressors, such as $-\mathrm{Fe},-\mathrm{N}$, and osmotic shock $(150 \mathrm{mM} \mathrm{NaCl})$, was also detected. Among these genes, CrMAPK3 is specifically functionally analyzed by RNA silencing.

\section{Results}

\section{Bioinformatics Analysis of MAPK Genes in Chlamydomonas}

Sixteen homologous genes (Table 1), which are localized in chromosomes 1, 2, 3, 8, 12, 13, 16, and 17, were identified by searching the Chlamydomonas genome database with Blast. The predicted open reading frames of these genes were $1062-5301 \mathrm{bp}$ in length, and their protein products contained 353-1766 amino acids with molecular weights of 39.8-178.76 kD and isoelectric points of 5.68-9.5. Fourteen of the MAPKs located in the cytosome were predicted by Euk-mPLoc2.0 except CrMAPK6 and CrMAPK14, which exist in the nucleus. Using PROSITE predictions, we verified that the 16 CrMAPKs were mitogen-activated protein kinases. Multi-sequence alignment of the MAPK-specific

Table 1 List of the 16 MAPK genes identified in C. reinhardtii and their sequence characteristics

\begin{tabular}{llllllll}
\hline Name & Locus Name & ORF (bp) & Amino Acids & kD & pl & Chromosomal localization & Sub cellular location \\
\hline CrMAPK2 & Cre08.g385050 & 2223 & 740 & 79.0 & 8.61 & chr8:4906426.4913322 F & Cytoplasm \\
CrMAPK3 & Cre12.g509000 & 1062 & 353 & 39.8 & 8.76 & chr12:2119590.2122035 R & Cytoplasm \\
CrMAPK4 & Cre17.g745447 & 2298 & 765 & 81.2 & 7.20 & chr17:6855222..6862749 F & Cytoplasm \\
CrMAPK5 & Cre13.g607300 & 1302 & 433 & 48.5 & 8.88 & chr13:5094976.5099387 F & Cytoplasm \\
CrMAPK6 & Cre12.g508900 & 1128 & 375 & 42.5 & 7.66 & chr12:2129460..2133325 R & Nuclear \\
CrMAPK7 & Cre16.g661100 & 1758 & 585 & 63.3 & 9.7 & chr16:2519399.2524020 F & Cytoplasm \\
CrMAPK8 & Cre01.g010000 & 1170 & 389 & 44.0 & 5.68 & chr1:1838122..1842287 F & Cytoplasm \\
CrMAPK9 & Cre12.g538300 & 1923 & 640 & 68.4 & 9.1 & chr12:6473633..6479228 F & Cytoplasm \\
CrMAPK10 & Cre01.g052800 & 3692 & 1163 & 117 & 9.91 & chr1:7342585..7351700 R & Cytoplasm \\
CrMAPK11 & Cre01.g052850 & 5199 & 1732 & 168 & 9.5 & chr1:7356808..7367075 F & Cytoplasm \\
CrMAPK12 & Cre03.g200200 & 5301 & 1766 & 178 & 9.36 & chr3:8184080..8192783 R & Cytoplasm \\
CrMAPK13 & Cre10.g432250 & 4752 & 1583 & 160 & 8.31 & chr10:1948371..1956866 R & Cytoplasm \\
CrMAPK14 & Cre03.g169500 & 3321 & 1106 & 114 & 9.49 & chr3:3709775.3716106 R & Nuclear \\
CrMAPK15 & Cre02.g111014 & 4212 & 1403 & 142 & 9.15 & chr2:5820048.5827789 F & Cytoplasm \\
CrMAPK16 & Cre17.g709500 & 3237 & 1078 & 107 & 7.93 & chr17:1790415..1798683 R & Cytoplasm \\
CrMAPK17 & Cre17.g709750 & 4956 & 1651 & 165 & 8.56 & chr17:1821974..1831590 F & Cytoplasm \\
\hline
\end{tabular}

$\mathrm{F}$ and $\mathrm{R}$ represent the forward and reverse directions on the chromosome, respectively. In total, 16 CrMAPK proteins were obtained by BLASTP search using the C. reinhardtii V5.5 proteome database and MAPK proteins from Arabidopsis thaliana as queries. The 16 CrMAPK genes were named based on their name annotated in JGI database. The molecular weights and pls of the 16 CrMAPK proteins were predicted using ExPASy. The CrMAPK sub-cellular locations were predicted using the Euk-mPLoc2.0 program 
TXY motifs in the CrMAPK proteins revealed that the $\mathrm{T}(\mathrm{D} / \mathrm{E} / \mathrm{T} / \mathrm{S} / \mathrm{P}) \mathrm{Y}$ activation loop motifs were conserved in the serine-threonine kinase (S-Tkc) domain in the 16 CrMAPKs (Fig. 1). CrMAPK3, CrMAPK6, and CrMAPK8 contain TEY; CrMAPK2, CrMAPK4, CrMAPK5, CrMAPK7, CrMAPK9, CrMAPK10, and CrMAPK13 comprise TDY; CrMAPK11, CrMAPK12, CrMAPK15, and CrMAPK16 possess TSY; CrMAPK17 is composed of TPY; and CrMAPK14 consists of TTY. Chlamydomonas MAPKs were divided into two groups by using MEGA6. Group I contained CrMAPK2 to CrMAPK10, whereas Group II comprised CrMAPK11 to CrMAPK17 (Fig. 2a). All of the MAPK genes contained 6 to 10 exons. The gene length ranged from $2.8 \mathrm{~kb}$ to $10.5 \mathrm{~kb}$. Among these genes, CrMAPK3 is the shortest and CrMAPK11 is the longest (Fig. 2b). In addition to the S-Tkc-conserved region, 8 other protein domains/ motifs, such as Syn N(Syntaxin N-terminal domain), RIO (RIO-like kinase), CUE (domain that may be involved in binding ubiquitin-conjugating enzymes), and Tyr-kc (tyrosine kinase catalytic domain) motifs (Fig. 2c), are present in CrMAPKs. The annotated CrMAPK1(Cre13.g582650) in JGI database is a small protein with 149 aa. After the alignment was compared with the other CrMAPKs(CrMAPK2CrMAPK17), the results revealed that the conserved domain (T(D/E/T/S/P)YXTRWYRAPEL(V)) in the MAPK family could not be found in CrMAPK1. As such, CrMAPK1 is not included in Table 1.

\section{Analysis of mRNA levels of MAPK gene under $-\mathrm{Fe},-\mathrm{N}$, and $150 \mathrm{mM} \mathrm{NaCl}$ stress conditions}

The RNA extracted from the samples of Chlamydomonas cultivated under $-\mathrm{Fe},-\mathrm{N}$, and $150 \mathrm{mM} \mathrm{NaCl}$ conditions was used for quantitative analysis, and the results are shown in Fig. 3. CrMAPK2-CrMAPK17 expression levels were affected by iron deficiency, nitrogen deficiency, and high salt concentration. Compared with the expression of the gene in the TAP medium, the mRNA expression levels of CrMAPK2, CrMAPK3, CrMAPK4, CrMAPK5, CrMAPK6, CrMAPK8, CrMAPK9, and $C r M A P K 11$ were increased by iron deficiency to various degrees. CrMAPK3, CrMAPK5, and CrMAPK11 respectively increased by $43-, 5-$, and 40 -fold after cultivation for $48 \mathrm{~h}$. However, iron deficiency decreased the mRNA levels of CrMAPK7, CrMAPK12, CrMAPK13, CrMAPK15, CrMAPK16, and CrMAPK17 after cultivation for $48 \mathrm{~h}$. In nitrogen deficiency, the mRNA expression levels of CrMAPK6 and CrMAPK14 were significantly increased, whereas the expression levels of most MAPKs, such as those of CrMAPK2, CrMAPK3, CrMAPK5, CrMAPK5, CrMAPK8, CrMAPK9, CrMAPK10, CrMAPK11, CrMAPK12, CrMAPK13, CrMAPK15, CrMAPK16, and CrMAPK17, of $C$. reinhardtii were inhibited, and the mRNA expression levels of these genes were significantly decreased. The mRNA expression levels of all MAPK genes were also inhibited under high salt $(150 \mathrm{mM} \mathrm{NaCl})$ condition, and the mRNA expression of CrMAPK17 was reduced by $10 \mathrm{E} 10$.

\section{CrMAPK3 positively regulates the expression of CrFOX1 gene}

The $C$. reinhardtii $2 \mathrm{~A} 38$ strain was prepared by using the integrated FOX1 promoter:ARS box into the chromosome of the CC425 strain. Under iron-deficient conditions, CrFOX1 promoted the ARS reporter expression and appeared deep blue when this gene was mixed with $\mathrm{XSO} 4$ substrate or yellow when this gene was mixed with $p$-nitrophenylsulfate. A total of 133 colonies were obtained after Maa7IR/CrMAPK3IR was transformed into $C$. reinhardtii $2 \mathrm{~A} 38$ and then transferred onto -

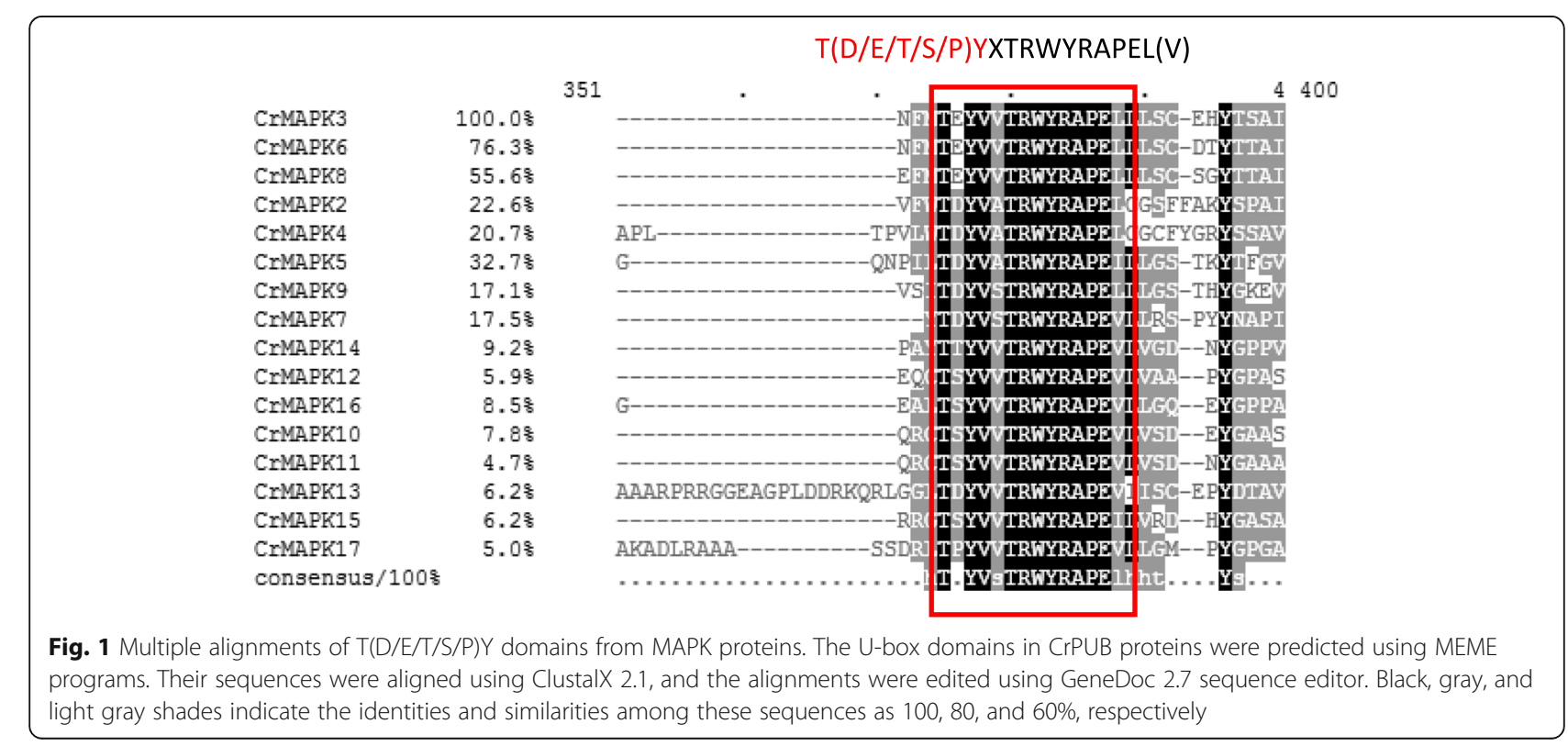




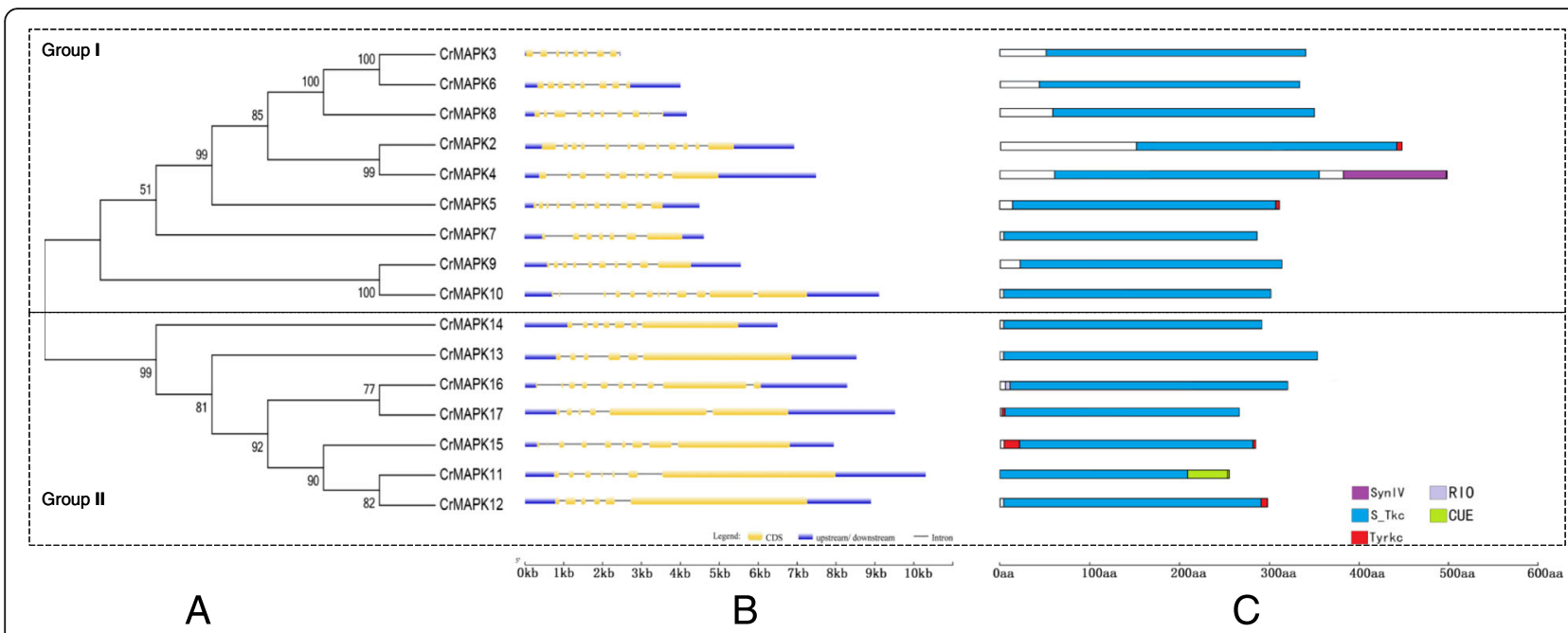

Fig. 2 An analytical view of the CrMAPK gene family. a An unrooted tree summarizing the evolutionary relationships among the 16 members of the CrMAPK family. Multiple alignments of the 16 CrMAPK protein sequences from C. reinhardtii were conducted using ClustalX 2.1. The phylogenetic tree was constructed using MEGA6. The numbers on each node are Shimodaira-Hasegawa-like test indices of statistical support provided by MEGA6. $\mathrm{Bar}=2.0$ is a branch length that represents the number of amino acid substitutions per site. The tree shows the 3 phylogenetic subfamilies (numbered I to ||l and marked with different color backgrounds) with high predictive values. $\mathbf{b}$ Intron/exon structures: the gene structures were drawn using the online tool GSDS. As shown in the legend, the exons and introns are indicated by green rectangles and thin lines, respectively. The untranslated regions (UTRs) are indicated by blue boxes. The sizes of exons and introns can be estimated using the scale shown at the bottom. c Schematic representation of the conserved motifs in the 16 CrMAPK proteins elucidated using SMART and PROSITE online. The different domains are indicated by different colored boxes denoted at the bottom right corner. The lengths of the proteins and motifs can be estimated using the scale shown at the bottom

Fe plates with XSO4 to determine the ARS activities. Only 44 colonies and the control sample of 2 A38 appeared blue, whereas the 99 other colonies were colorless or light blue. Furthermore, $74.4 \%$ of chromogenic reactions indicated that CrMAPK3 silencing affected the FOX1 promoter function in -Fe. The transformants of RNAi11, RNAi37, and RNAi62 appeared colorless in both + Fe $(16 \mathrm{uM})$ and $-\mathrm{Fe}$ except the non-transgenic C. reinhardtii 2A38 control, which appeared deep blue under $-\mathrm{Fe}$ conditions (Fig. 4a). These results were further confirmed by the ARS activity in transgenic strains. The ARS activities of RNAi11, RNAi37, and RNAi62 respectively decreased by 82,85 , and $83 \%$ compared with those of the C. reinhardtii 2A38 control (Fig. $4 \mathrm{~b}$ ). The mRNA of the ARS2 of the transgenic stains decreased by more than $97 \%$ in $-\mathrm{Fe}$ (Fig. 4c). The mRNA levels of the target CrMAPK3 of the transgenic strains RNAi11, RNAi37, and RNAi62 decreased remarkably by 97,97 , and $98 \%$, respectively (Fig. 4 d). These data implied that CrMAPK3 silencing positively regulated FOX1:ARS expression. CrMAPK3 silencing also decreased the gene expression of endogenous FOX1 in Chlamydomonas. The mRNA levels of CrFOX1 of the RNAi11 strain decreased by 63,54 , and $71 \%$ when this strain was cultured for 12,24 , and $48 \mathrm{~h}$ under the $-\mathrm{Fe}$ condition, respectively. CrMAPK3 silencing also repressed the iron-induced upregulation of CrFOX1 gene expression. Therefore, CrMAPK3 positively regulated the endogenous expression of $\mathrm{CrFOX1}$ (Fig. 4e).

\section{CrMAPK3 positively regulates the expression levels of} iron uptake-associated genes

Chlamydomonas exhibits an iron uptake pattern similar to Type I plants. During iron deficiency, Chlamydomonas cells undergo the affinity iron absorption mechanism by inducing the expression of CrFOX1 [19], CrNRAMP2 [18], CrATX1 [22], CrFTR1 [20], and CrFEA1 to enhance iron absorption [18]. CrFEA1 is located in the cell walls and responsible for the transport of reduced $\mathrm{Fe}^{2+}$ via FRE1 (homolog of Arabidopsis FRO2) to CrFOX1, which is found in the plasma membrane (homolog of yeast FET3 [23]), and reoxidizes $\mathrm{Fe}^{2+}$ to $\mathrm{Fe}^{3+}$. CrFOX1 is then transported inside the cells through the plasma membrane protein CrFTR1, and ATX1 of yeast transports $\mathrm{Cu}^{2+}$ to the cytoplasm. Thus far, direct evidence supporting iron transmission has yet to be obtained, but studies have shown that ATX1 is an iron-deficiency-inducible protein. The NRAMP gene family is located on the vacuole membrane, and it shuttles $\mathrm{Fe}^{2+}$ between vacuole membranes to maintain the iron concentration in the cytoplasm. The mRNA levels of iron absorption-related genes, such as CrNRAMP2, CrATX1, CrFTR1, and CrFEA1, in the CrMAPK3 RNAi transgenic strain RNAil1 are shown in Fig. 5. The mRNA levels of the genes decreased after the strains were cultivated for $48 \mathrm{~h}$ under $-\mathrm{Fe}$. The mRNA level of CrNRAMP2 was decreased by $86 \%$ compared with the control after cultivation for $48 \mathrm{~h}$ in $-\mathrm{Fe}$. Similarly, the mRNA levels of CrATX1, CrFTR1, and CrFEA1 were 


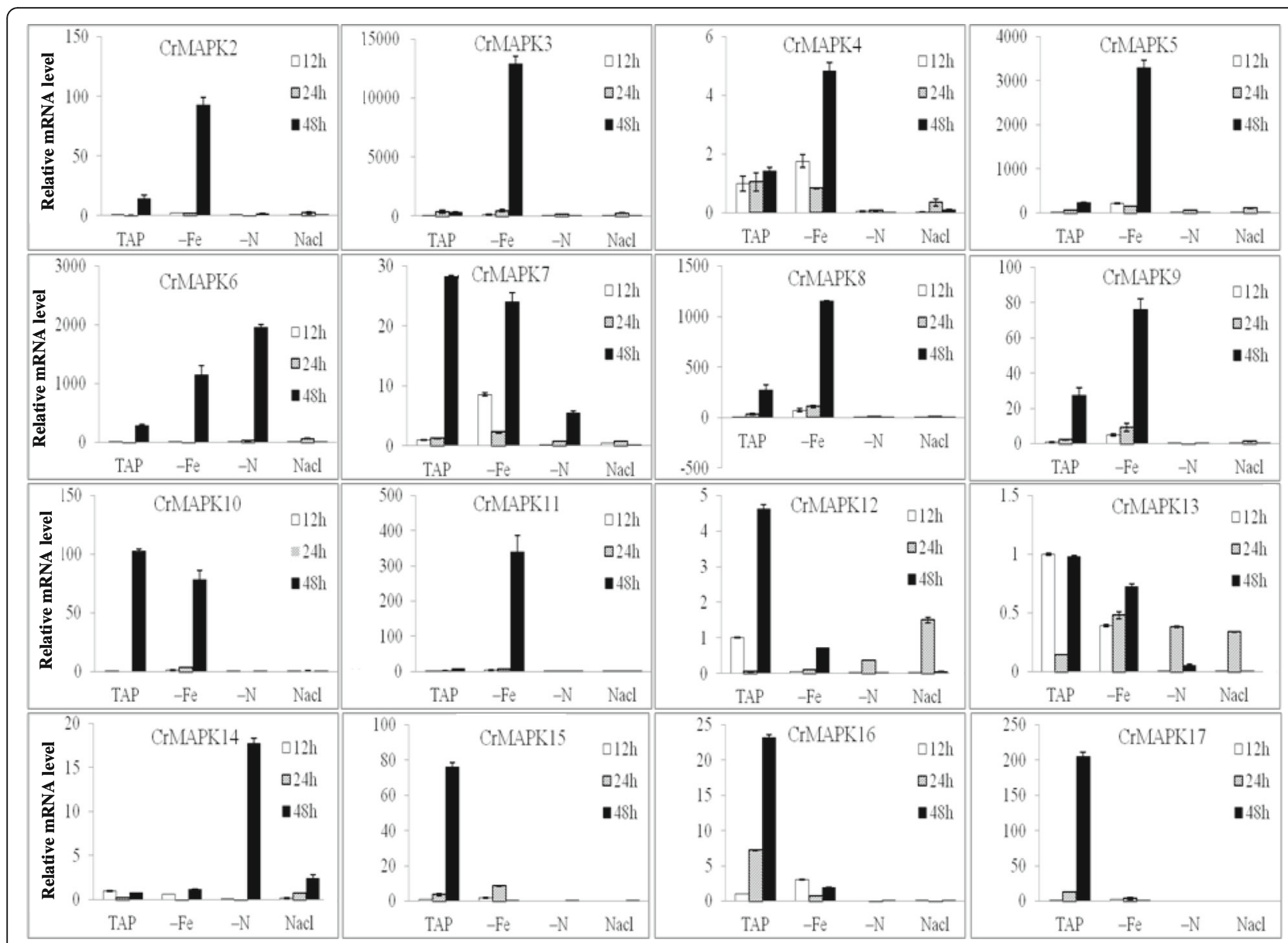

Fig. 3 Results of qPCR analysis of the CrMAPK genes under - Fe, $-\mathrm{N}$, or $150 \mathrm{mM} \mathrm{NaCl}$ conditions. C. reinhardtii CC425 was pre-cultured in TAP to the mid-logarithmic phase, followed by centrifugation and resuspension in TAP, TAP-Fe, TAP-N, and TAP with $150 \mathrm{mM} \mathrm{NaCl}$. All the samples continued culturing for 12,36, and $48 \mathrm{~h}$. The cells were collected, and RNA samples were isolated. The gene transcript levels were determined using Real Time quantitative PCR. All expression values were normalized to the value of the 185 rRNA gene. The relative amounts were calibrated based on the number of transcripts of the corresponding genes in cells maintained in TAP for $12 \mathrm{~h}$

decreased by 96,96 , and approximately $53 \%$, respectively. These results indicated the association of CrMAPK3 of Chlamydomonas with the iron metabolism-related genes. In the CrMAPK3-silenced strain RNAi11, the mRNA levels of the genes, including CrNRAMP2, CrATX1, CrFTR1, and CrFEA1, were also decreased when the mRNA level of CrMAPK3 was decreased. Thus, CrMAPK3 might positively regulate the expression of iron-uptake-associated genes, such as CrNRAMP2, CrATX1, CrFTR1, and CrFEA1.

\section{Discussion}

MAPKs are widely distributed in eukaryotic organisms, such as yeast, humans, and plants, and are involved in phosphorylation signaling cascades in extracellular amplification and intracellular transduction [23]. The MAPK pathway is responsive to biological and non-biological stress stimuli, hormones, or growth factors and to cell division and apoptosis. Moreover, the MAPK pathway comprises MAPKKK, MAPKK, and MAPK and amplifies signals via subsequent phosphorylation by using protein kinases and by migrating to the nucleus; thus, the extracellular stimuli of membrane receptors are connected to the molecular effectors of the cytoplasm and the nucleus [24, 25]. A few MAPKs, including 20 in Arabidopsis, 17 in rice, 19 in corn, 21 in aspen (Populus), 17 in tobacco, 16 in tomato, and 26 in apple, have been identified [26-28]. Proteins encoded by MAPKs in different species contain various domains. In Chlamydomonas, 3 of TEY, 7 of TDY, 4 of TSY, 1 of TPY, and 1 of TTY exist. In Arabidopsis, 8 of TDY and 12 of TEY are present. These diversities of types and kinase domains demonstrate that MAPKs participate in many metabolic activities. Through cluster analysis, we found that TDY and TEY of Chlamydomonas kinases are highly homologous to those of Arabidopsis kinases possibly because only TDY and TEY domains are found in Arabidopsis. Other domains are highly similar to human kinases.

Organisms need iron for respiration, DNA synthesis, and enzyme reactions. Transport systems have been developed 

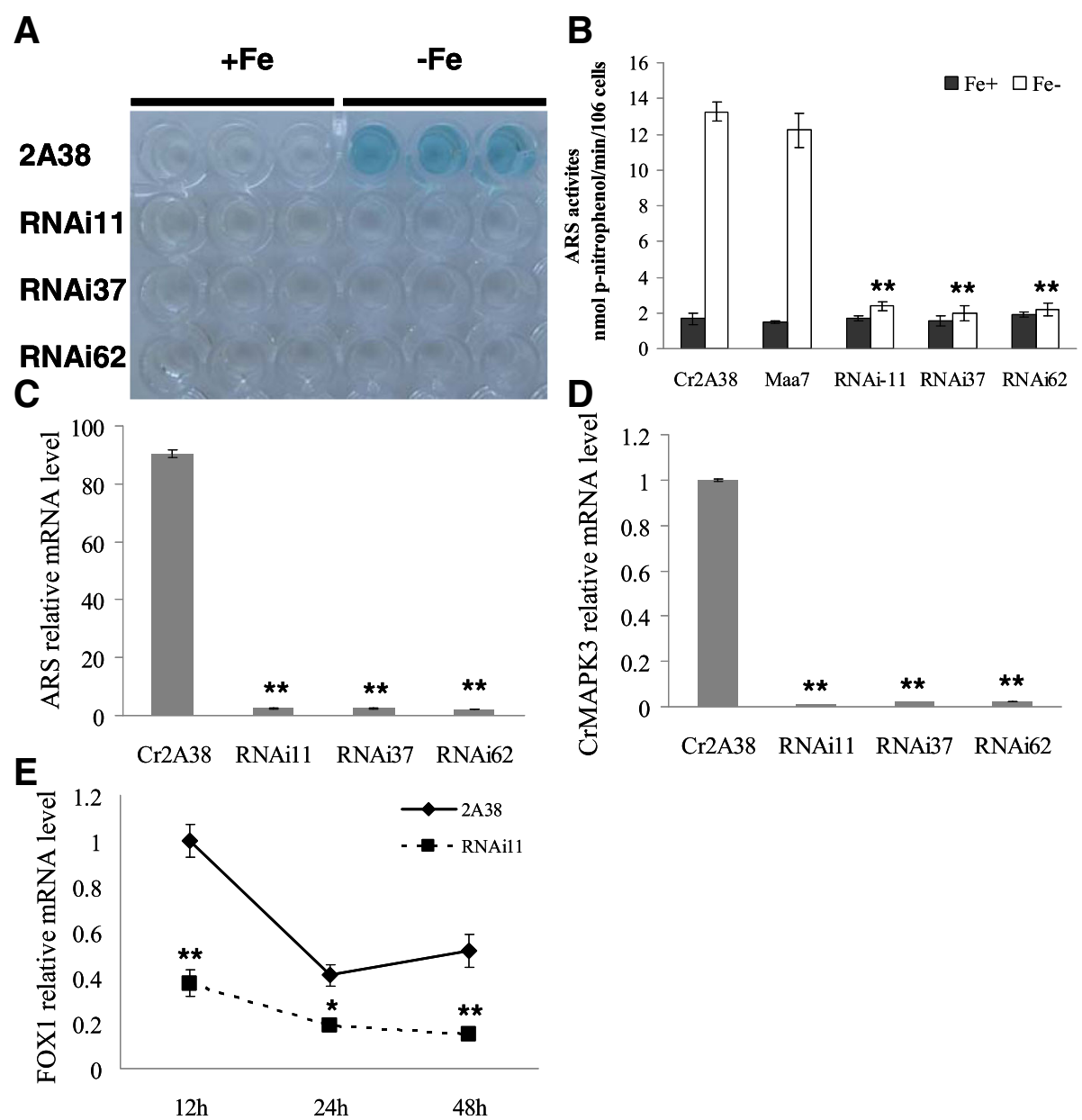

Fig. 4 Analysis of CrMAPK3 RNAi transgenic algal strains. Of the 133 CrMAPK3 RNAi transformants, 99 were colorless or light blue. Among them, the ARS activities of transgenic strains RNAi11, RNAi37, and RNAi62 were significantly decreased under - Fe (a, b). Moreover, the mRNA levels of ARS were significantly decreased (c). The mRNA level of target gene CrMAPK3 was decreased by 97-98\% compared with the control (d), indicating that CrMAPK3 in transgenic strains of RNAi11, RNAi37, and RNAi62 has been effectively silenced. The mRNA levels of endogenous CrFOX1 were reduced by 63,54 , and $71 \%$, respectively, at 12, 24, and $48 \mathrm{~h}$ post-incubation in -Fe, indicating that CrMAPK3 positively regulates the expression of CrFOX1 gene (e). The data are shown as the means $( \pm \mathrm{SD}, n=3)$. Significance is indicated as ${ }^{*} P<0.05,{ }^{* *} P<0.01$

for iron absorption because iron balance is vital. Iron regulation, especially iron absorption and transportation, has been extensively investigated, but iron signal response systems have been rarely explored. Iron deficiency in humans causes iron deficiency anemia and adolescent iron deficiency 1 syndrome. Iron is an important element required by the body; excessive or scarce amounts of iron likely cause metabolic disorders; therefore, organisms should have a sophisticated control system to regulate the dynamic balance of iron elements [16].

Iron deficiency is the third-most important limiting factor of plant growth and yield in agriculture. Photosynthetic plants reduce their chlorophyll synthesis and photosynthesis rate under iron-deficient conditions.

In humans, iron deficiency causes anemia. Conversely, excess iron increases the risk of liver disease, heart attack, and hypothyroidism. Iron is also a limiting factor in the growth of some tumor cells, and iron chelators are used clinically to inhibit tumor cell growth. Furthermore, studies on iron MAPK signal cascades have focused on human cancers. Iron deficiency inhibits the mitosis of lung carcinoma cells, melanoma cells, and dysembryoplastic neuroepithelial tumor cells and thus induces cell apoptosis [29-31]. Therefore, iron chelators, desferrioxamine (DFO), and $\mathrm{Dp} 44 \mathrm{mT}$ are used to treat these cancers clinically [32, 33]. Iron deficiency signals are also transduced through the activation of JNA and P38 by ASK1 (MAPKKK) to regulate the suspension of the mitotic activity and apoptosis of cancer cells [34].

Plant MAPK gene responses to various stresses have also been detected. In our study, gene expression analysis revealed that 16 MAPK genes in Chlamydomonas were involved in response to stress. During iron deficiency, 8 MAPK genes, including CrMAPK3, were upregulated. 

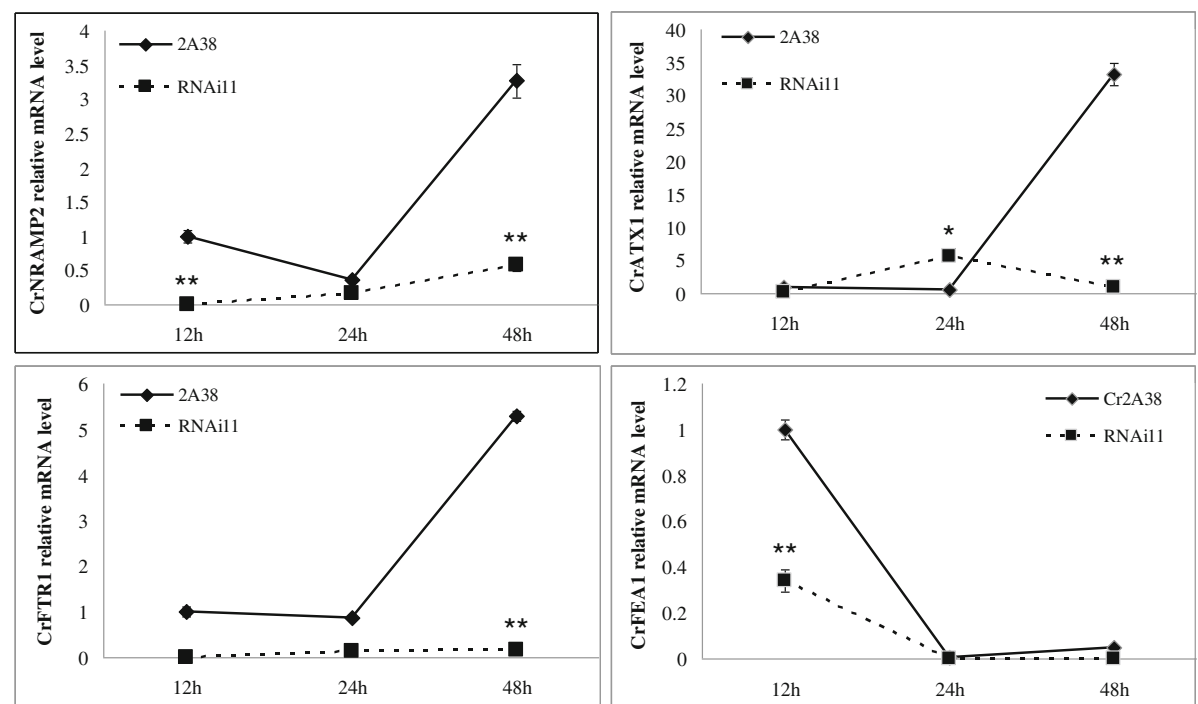

Fig. 5 mRNA expressions of iron absorption-related genes in CrMAPK3 RNAi transgenic strain RNAi11 in -Fe. In the CrMAPK3-silenced strain RNAi1 1, the mRNA levels of genes, including CrNRAMP2, CrATX1, CrFTR1, and CrFEA1, were decreased when the mRNA level of CrMAPK3 was decreased and after they were incubated with -Fe after $48 \mathrm{~h}$. This finding indicated that CrMAPK3 may positively regulate the expression of iron uptake-associated genes, such as CrNRAMP2, CrATX1, CrFTR1, and CrFEA1. The data are shown as means \pm SD $(n=3)$. Significance is indicated as ${ }^{*} P<0.05,{ }^{* *} P<0.01$

Therefore, CrMAPK3 possibly responded to iron regulation. These findings were further verified by silencing CrMAPK3, and our results demonstrated that the mRNA levels of FOX1-ARS, the enzyme activities of ARS, and the endogenous mRNA level of CrFOX1 decreased. Therefore, $C r M A P K 3$ positively regulated $C r F O X 1$ expression. The mRNA levels of $-\mathrm{Fe}$-inducing genes, including CrNRAMP2, CrATX1, CrFTR1, and CrFEA1, and the expression of $C r M A P K 3$ were reduced. These findings confirmed that CrMAPK3 positively regulated the expression of iron-absorption genes. However, the exact proteins upstream and downstream of CrMAPK3 should be identified to reveal the MAPK pathway of iron deficiency response in Chlamydomonas.

\section{Methods}

\section{Algal strains and culture conditions}

C. reinhardtii CC425 (mt) was purchased from the Chlamydomonas Genetics Center at Duke University. C. reinhardtii $2 \mathrm{~A} 38$ is a transgenic strain with an integrated Fox1 promoter:ARS chimeric gene in C. reinhardtii CC425 genome. Under iron-deficient conditions, the CrFOX1 promoter in 2A38 strain stimulated the ARS gene expression and appeared blue when the XSO4 substrate was added. Liquid cultures were grown in the TAP medium at $26^{\circ} \mathrm{C}$ with agitation at $220 \mathrm{rpm}$ under $110 \mu \mathrm{mol} \cdot \mathrm{m}^{-2} \mathrm{~s}^{-1}$ of continuous light for 3 days and then to the TAP, TAP-Fe, TAP-N, or TAP $+150 \mathrm{mM} \mathrm{NaCl}$ media for various time periods $(12,24$, and $36 \mathrm{~h})$. Total RNA was extracted to prepare cDNA for gene cloning and real-time PCR assay.
All Chlamydomonas strains were cultured in the TAP or deficiency medium of TAP with Hunter's trace element mix.

\section{Bioinformatics analysis of MAPK gene family of Chlamydomonas}

Chlamydomonas MAPK homologous genes were retrieved from Chlamydomonas database (https://phytozome.jgi.doe.gov/pz/portal.html) by using the BLAST of Arabidopsis MAPK. Multiple sequence alignments were generated using ClustalX 2.1 and MEGA6. The following parameters were predicted: molecular weights and isoelectric points of proteins in Expasy (http://web.expasy.org/compute_pi/); protein structures in SMART; and conserved protein motifs in PROSITE (http://prosite.expasy.org/) and MEME (http://meme.nbcr.net/meme/). The structures of CrMAPK genes were generated online by using the Gene Structure Display Server (GSDS) (http:// gsds.cbi.pku.edu.cn/), and the homologous chromosome segments were detected using a synteny plot in Plaza (http://bioinformatics.psb.ugent.be/plaza/versions/picoplaza/synteny/index). The CrMAPK genes were subjected to BLAST analysis in Plaza, and their duplication patterns were detected using a synteny plot. The subcellular localization of Chlamydomonas MAPKs was performed using Euk-mPLoc2.0 (http://www.csbio.sjtu.edu.cn/bioinf/ euk-multi-2/).

\section{Statistical analyses}

Data were presented as mean \pm S.D. One-way ANOVA followed by Duncan's post-test was performed to examine 
significant differences between means. In all cases, comparisons showing $P<0.05$ were considered significant.

\section{mRNA abundance detection}

Three independent cell populations exposed to different stress conditions in various periods were collected. Total RNA was extracted and real-time PCR was performed as described previously [35]. The primers used in this study are listed in Tables 2 and 3 . All the products were $300+/$ $50 \mathrm{nt}$ The amplification rate of each transcript $(\mathrm{Ct})$ was calculated using the PCR Base Line Subtracted method performed in the iCycler software at a constant fluorescence

Table 2 Primer sequences for amplifying the Chlamydomonas MAPK genes

\begin{tabular}{|c|c|}
\hline Primer Name & Primer Sequences \\
\hline CrMAPK2-F & GAGGCAAACCGATACACGAT \\
\hline CrMAPK2-R & CGAGTACTTGGCGAAGAAGG \\
\hline CrMAPK3-F & CGTCCGCAAAAGACAGTGTA \\
\hline CrMAPK3-R & GGAGCACCTGGTAGACGAAG \\
\hline CrMAPK4-F & GCACAGCCTCATAGGAAAGG \\
\hline CrMAPK4-R & CCAATCACTGTGTGCAGGTC \\
\hline CrMAPK5-F & GGAGGTGCACAAGCAGTACA \\
\hline CrMAPK5-R & GTGCTACCCAGCAGGATCTC \\
\hline CrMAPK6-F & CCAGCTCAAGCTCATCATCA \\
\hline CrMAPK6-R & CATCTCGTCAAAGTCCAGCA \\
\hline CrMAPK7-F & AAGACCGGGACAAGTTCCTT \\
\hline CrMAPK7-R & ATGTACAGCTCCGCCATGAT \\
\hline CrMAPK8-F & AGAGATTTGAAGCCCAGCAA \\
\hline CrMAPK8-R & ACCTTGGTGATGAGGGACAG \\
\hline CrMAPK9-F & GCTGTGCGACTTTGGCTTTGC \\
\hline CrMAPK9-R & TACTTGCGGTCCAGCGTCTCCG \\
\hline CrMAPK10-F & CGTTGTGGCGGTGAAAGG \\
\hline CrMAPK10-R & GAACCCGAAGTCGCACAGC \\
\hline CrMAPK11-F & ATCAAGCCCGCCAACATCC \\
\hline CrMAPK11-R & GTTGTCAGATACCAGCACCTCC \\
\hline CrMAPK12-F & GATGCCTTCAAATCTAAAACCG \\
\hline CrMAPK12-R & AAGTCGCACAGCCGCACCAC \\
\hline CrMAPK13-F & TCGGGCACGCCGCTGTTC \\
\hline CrMAPK13-R & TAGCGTGCGACCTGGTGCGG \\
\hline CrMAPK14-F & GGGCGTATGGGTCGGTAT \\
\hline CrMAPK14-R & GCAGGTTGACGATGTTCAC \\
\hline CrMAPK15-F & CACGATTGATGCGATAGAGGAG \\
\hline CrMAPK15-R & AAGGTCGGGAACGGTGGA \\
\hline CrMAPK16-F & TGGCAGTTAGCCTCCGTCATT \\
\hline CrMAPK16-R & CGCAAAGCCAAAGTCGCA \\
\hline CrMAPK17-F & GAGGTTACCGTGCTCAATGGC \\
\hline CrMAPK17-R & GGGTAGCGGTCCTCCAACA \\
\hline
\end{tabular}

Table 3 Primer sequences for amplifying Chlamydomonas iron responsive genes

\begin{tabular}{llll}
\hline $\begin{array}{lll}\text { Gene } \\
\text { Name }\end{array}$ & Locus Name & $\begin{array}{l}\text { Primer } \\
\text { Name }\end{array}$ & Primer Sequences \\
\hline CrARS2 & Cre16.g671350 & ARS2-F & ATGGGTGCCTCGCGGTGTC \\
& & ARS2-R & GTAGCGGATGTACTTGGCAG \\
CrFOX1 & Cre09.g393150 & FOX1-F & GACGTGGAGGCCCAGAAG \\
& & FOX1-R & CGCGACGAAGTAGGTGTG \\
CrFTR1 & Cre03.g192050 & FTR1-F & TCTTCGGGAGACCATGAG \\
& & FTR1-R & GAAGCATAGCAAAGCCAAGG \\
CrFEA1 & Cre12.g546550 & FEA1-F & CTCAAGTACCACCTGCACGA \\
& & FEA1-R & ACATAGCTCTTGCGAGGAA \\
CrATX1 & Cre09.g392467 & ATX1-F & AGCTCGTGTCCTCGTAAAGC \\
& & ATX1-R & CTGCAACAGGTCCGTGTAA \\
CrNRAMP2 & Cre07.g321951 & NRAMP2-F & CTGTCGCAGGTGATCCTGT \\
& & NRAMP2-R & TITGCACCACCAGGTAATG \\
\hline
\end{tabular}

level. Cts were determined with three repeats. Relative fold differences were calculated on the basis of the relative quantification analytical method $\left(2^{-\Delta \Delta C T}\right)$ using $18 \mathrm{~s}$ rRNA amplification as internal standard [36].

\section{Construction of CrMAPK3 RNA interference vector and transformation of Chlamydomonas}

Using Chlamydomonas cDNA as a template, we amplified the fragments through PCR with forward primer CrMAPK3-F: CGTCCGCAAAAGACAGTGTA and reverse primer CrMAPK3-R: CTTCGTCTACCAGGTGC TCC. We then inserted the amplified fragments into pMD18-T vector to generate CrMAPK3-18 T, which was further digested with HindIII and BamHI and ligated into the intermediate vector T282 to produce CrMAPK3T282. CrMAPK3-T282-CrMAPK3 with inverted repeat sequence of CrMAPK3 (CrMAPK3IR) was developed by digesting CrMAPK3-18 T and CrMAPK3-T282. CrMAP $K 3 I R$ was inserted into EcoRI-digested pMaa7/XIR to produce Maa7IR/CrMAPK3IR. Maa7IR/CrMAPK3IR was then transformed into $C$. reinhardtii $2 \mathrm{~A} 38$ by applying the glass bead procedure [37].

\section{ARS (arylsulfatase) activity detection}

ARS activity was determined as described by Davies and Grossman [38]. XSO4 (10 mM) was added to plates with Fe TAP solid medium and scribed before clones were inoculated. After 1 day, the transformants that expressed ARS activity were identified using blue halos around their colonies. The cells were initially collected by centrifugation to quantify the ARS activity. The supernatant was mixed with $0.1 \mathrm{M}$ glycine- $\mathrm{NaOH}$ at $\mathrm{pH}$ 9.0, $10 \mathrm{mM}$ imidazole, and $4.5 \mathrm{mM} p$-nitrophenyl sulfate. The reaction mixture was incubated at $27{ }^{\circ} \mathrm{C}$ for $30 \mathrm{~min}$. The reaction was terminated by adding $0.25 \mathrm{M} \mathrm{NaOH}$, and its absorbance at $410 \mathrm{~nm}$ 
was determined. A standard curve of $p$-nitrophenol (Sigma Chemical Co.) was obtained using $0.2 \mathrm{M} \mathrm{NaOH}$.

\section{Conclusions}

Silencing CrMAPK3 decreased the mRNA levels and ARS activities of FOX1:ARS chimeric gene and endogenous CrFOX1. The mRNA levels of iron-responsive genes, such as CrNRAMP2, CrATX1, CrFTR1, and CrFEA1, were also remarkably reduced. Therefore, CrMAPK 3 regulated the expression of iron-deficiency-responsive genes in C. reinhardtii.

\section{Acknowledgments}

Not applicable.

\section{Funding}

This study was supported by the National Natural Science Foundation of China (31160050, 31360051), the Funds of Hainan Engineering and Technological Research (GCZX2011006, GCZX2012004, GCZX2013004), and the Key Projects of Hainan Province (ZDYF2016021).

\section{Availability of data and materials}

All of the data generated or analyzed in this study are included in this published article.

\section{Authors' contributions}

XW Fei and XD Deng designed experiments. XW Fei and JM Yu performed experiments. JM Yu and YJ Li analyzed data. XW Fei and XD Deng wrote the manuscript. All authors read and approved the final manuscript.

\section{Competing interest}

The authors declare that they have no competing interests.

\section{Consent for publication}

Not applicable.

\section{Ethics approval and consent to participate}

No human subjects or animals were used in this study.

\section{Publisher's Note}

Springer Nature remains neutral with regard to jurisdictional claims in published maps and institutional affiliations.

\section{Author details}

'School of Science, Hainan Medical College, Haikou 571101, China. ${ }^{2}$ Institute of Tropical Bioscience and Biotechnology, Chinese Academy of Tropical Agricultural Science, Key Laboratory of Tropical Crop Biotechnology, Ministry of Agriculture, Haikou 571101, China.

Received: 11 January 2017 Accepted: 28 April 2017

Published online: 16 May 2017

\section{References}

1. Fei XW, Deng XD. A novel Fe deficiency responsive element (FeRE) regulates the expression of atx 1 in Chlamydomonas reinharditii. Plant Cell Physiol. 2007;48:1496-503.

2. Mohanta KT, Arora PK. Identification of new members of the MAPK gene family in plants shows diverse conserved domains and novel activation loop variants. BMC Genomics. 2015;6:16-58.

3. Besteiro MAG, UIm R. Phosphorylation and stabilization of arabidopsis MAP kinase phosphatase 1 in response to UV-B stress. J Biol Chem. 2013;288:480-6.

4. Caunt CJ, Keyse SM. Dual-specificity MAP kinase phosphatases (MKPs)Shaping the outcome of MAP kinase signalling. FEBS J. 2013;280:489-504.

5. Rodriguez MC, Petersen M, Mundy J. Mitogen-activated proteinkinase signaling in plants. Annu Rev Plant Biol. 2010;61:621-49.

6. Nakagami $H$, Pitzschke A, Hirt H. Emerging MAP kinase pathways in plant stress signalling[J]. Trends Plant Sci. 2005;10:1360-85.
7. Mizoguchi T, Hayashida N, Yamaguchi-Shinozaki K. ATMPKs: A gene family of plant MAP kinases in Arabidopsis thaliana. FEBS Lett. 1993:336:440-4.

8. Lieberherr D, Thao NP, Nakashima A. A sphing olipid elicitor-inducible mitogen-activated protein kinase is regulated by the small GTPase OsRac1 and heterotrimeric G-protein in rice. Plant Physiol. 2005;138:1644-52.

9. Lalle M, Visconti S, Marra M. ZmMPK6, a novel maize MAP kinase that interacts with 14-3-3 proteins. Plant Mol Biol. 2005;59:713-22.

10. Takezawa D. Elicitor- and A23187-induced expression of WCK-1, a gene encoding mitogen-activated protein kinase in wheat. Plant Mol Biol. 1999; 40:921-33.

11. Knetsch M, Wang M, Snaar-Jagalska BE. Abscisic acid induces mitogenactivated protein kinase activation in barley aleuron protoplasts. Plant Cell. 1996:8:1061-7.

12. Huttly AK, Phillips AL. Gibberellin-regulated expression in oataleurone cells of two kinases that show homology to MAP kinase and a ribosomal protein kinase. Plant Mol Biol. 1995;27:1043-52.

13. Wilson C, Anglmayer R, Vicente O. Molecular cloning, functional expression in Escherichia coli, and characterization of multiple mitogen-activated protein kinases from tobacco. Eur J Biochem. 1995:233:249-57.

14. Jonak C, Ökrész L, Bögre L. Complexity, cross talk and integration of plant MAP kinase signaling. Curr Opin Plant Biol. 2002;5:415-24.

15. Singh $\mathrm{R}$, Lee $\mathrm{MO}$, Lee JE. Rice mitogen-activated protein kinase interactome analysis using the yeast two-hybrid system. Plant Physiol. 2012;160:477-87.

16. Deng XD, Eriksson M. Two iron-responsive promoter elements control expression of FOX1 in Chlamydomonas reinhardtii. Eukaryot Cell. 2007;6:2163-7.

17. Robinson NJ, Procter CM, Connolly EL, Guerinot ML. A ferric-chelate reductase for iron uptake from soils. Nature. 1999;397:694-7.

18. Allen MD, del Campo JA, Kropat J, Merchant SS. FEA1, FEA2, and FRE1, encoding two homologous secreted proteins and a candidate ferrireductase, are expressed coordinately with FOX1 and FTR1 in irondeficient Chlamydomonas reinhardtii. Eukaryot Cell. 2007:6:1841-52.

19. Chen JC, Hsieh SI, Kropat J, Merchant SS. A ferroxidase encoded by FOX1 contributes to iron assimilation under conditions of poor iron nutrition in Chlamydomonas. Eukaryot Cell. 2008;7:541-5.

20. La Fontaine S, Quinn JM, Nakamoto SS, Page MD, Göhre V, Moseley JL, Kropat J, Merchant SS. Copper-dependent iron assimilation pathway in the model photosynthetic eukaryote Chlamydomonas reinhardtii. Eukaryot Cell. 2002;1:736-57.

21. Terzulli A, Kosman DJ. Analysis of the high-affinity iron uptake system at the Chlamydomonas reinhardtii plasma membrane. Eukaryot Cell. 2010;9:815-26.

22. Pufahl RA, Singer CP, Peariso KL, Lin SJ, Schmidt PJ, Fahrni CJ, Culotta VC, Penner-Hahn JE, O'Halloran TV. Metal ion chaperone function of the soluble Cu (I) receptor Atx1. Science. 1997;278:853-6.

23. Liang W, Yang B, Yu BJ, Zhou Z, Li C, Jia M. Identification and analysis of MKK and MPK gene families in canola (Brassica napus L.). BMC Genomics. 2013;14:392

24. Chen L, Hu W, Tan S, Wang M, Ma Z, Zhou S. Genome-wide identification and analysis of MAPK and MAPKK gene families in Brachypodium distachyon. PLoS One 2012:7:e46744

25. Janitza P, Ullrich KK, Quint M. Toward a comprehensive phylogenetic reconstruction of the evolutionary history of mitogen-activated protein kinases in the plant kingdom. Front Plant Sci. 2012;3:1-11.

26. Zhang S, Xu R, Luo X, Jiang Z, Shu H. Genome-wide identification and expression analysis of MAPK and MAPKK gene family in Malus domestica. Gene. 2013:531:377-87.

27. Nicole MC, Hamel LP, Morency MJ. MAP-ping genomic organization and organ-specific expression profiles of poplar MAP kinases and MAP kinase kinases. BMC Genom. 2006;7:223

28. Kong F, Wang J, Cheng L. Genome-wide analysis of the mitogen-activated protein kinase gene family in Solanum lycopersicum. Gene. 2012;499:108-20.

29. Le NTV, Richardson DR. The role of iron in cell cycle progression and the proliferation of neoplastic cells. Biochim Biophys Acta. 2002;1603:31-46.

30. Brodie C, Siriwardana G, Lucas J, Schleicher R, Terada N, Szepesi A, Gelfand E, Seligman P. Neuroblastoma sensitivity to growth inhibition by deferrioxamine: evidence for a block in G1 phase of the cell cycle. Cancer Res. 1993:53:3968-75.

31. Hileti D, Panayiotidis P. Hoffbrand AV, Iron chelators induce apoptosis in proliferating cells. Br J Haematol. 1995;89:181-7.

32. Buss JL, Torti FM, Torti SV. The role of iron chelation in cancer therapy. Cur Med Chem. 2003;10:1021-34. 
33. Kalinowski DS, Richardson DR. The evolution of iron chelators for the treatment of iron overload disease and cancer. Pharmacol Rev. 2005;57:547-83.

34. Yu Y, Richardson D. Cellular iron depletion stimulates the JNK and p38 MAPK signaling transduction pathways, dissociation of ASK1-thioredoxin, and activation of ASK1. J Biol Chem. 2011;286:15413-27.

35. Luo Q, Li Y, Wang W, Fei X, Deng X. Genome-wide survey and expression analysis of Chlamydomonas reinhardtii U-box E3 ubiquitin ligases (CrPUBs) reveal a functional lipid metabolism module. PLoS One. 2015;10:e0122600

36. Livak KJ, Schmittgen TD. Analysis of relative gene expression data using real-time quantitative PCR and the 2(-Delta Delta C(T)) Method. Methods. 2001;25:402-8

37. Kindle KL. High frequency nuclear transformation of Chlamydomonas reinhardtii. Proc Natl Acad Sci U S A. 1990;87:1228-32.

38. Davies JP, Grossman AR. Sequences controlling transcription of the Chlamydomonas reinhardtii beta 2-tubulin gene after deflagellation and during the cell cycle. Mol Cell Biol. 1994;14:5165-74.

Submit your next manuscript to BioMed Central and we will help you at every step:

- We accept pre-submission inquiries

- Our selector tool helps you to find the most relevant journal

- We provide round the clock customer support

- Convenient online submission

- Thorough peer review

- Inclusion in PubMed and all major indexing services

- Maximum visibility for your research

Submit your manuscript at www.biomedcentral.com/submit
Biomed Central 